\title{
IDENTIDADE PROFISSIONAL DOS ENFERMEIROS DE SERVIÇOS DE SAÚDE MUNICIPAL
}

Carmem Lúcia Colomé Beck ${ }^{1}$, Francine Cassol Prestes², Juliana Petri Tavares ${ }^{3}$, Rosângela Marion da Silva ${ }^{4}$, Adelina Giacomelli Prochonow ${ }^{5}$, Carolina de Quadros Nonnenmacher ${ }^{6}$

RESUMO: Este estudo objetiva apresentar a percepção dos enfermeiros dos Serviços de Saúde do Rio Grande do Sul acerca da sua identidade profissional. Trata-se de uma pesquisa qualitativa, com a utilização de um questionário para a coleta dos dados. Participaram 29 enfermeiros dos Serviços de Saúde.Para a análise dos dados foi utilizada a análise temática e os mesmos organizados em torno de três eixos: auto-imagem, heteroimagem e imagem da realidade. Como resultados, os enfermeiros se percebem como cuidadores resolutivos, úteis e necessários na assistência. Sentem-se pouco reconhecidos por outros profissionais e identificam uma visão inespecífica do enfermeiro por parte dos usuários. Os enfermeiros estão inseridos em um contexto complexo e identificam o reconhecimento da comunidade, apesar da falta de condições para realizar seu trabalho. Portanto, a identidade profissional do enfermeiro é formada por reflexos da autoimagem, heteroimagem e imagem da realidade consubstanciados.

PALAVRAS-CHAVE: Papel do profissional de enfermagem; Competência profissional; Saúde do trabalhador.

\section{PROFESSIONAL IDENTITY OF NURSES OF A COUNTY HEALTH SERVICE}

ABSTRACT: This study aims to present the perception of nurses from the Health Services in the State of Rio Grande do Sul regarding their professional identity. It is a qualitative research using a survey for data collection. A total of 29 nurses that worked in Health Services. In order to collect data, a thematic analysis was used and organized into three axes: selfimage, hetero-image, and image from reality. The results display the fact that nurses consider themselves as resolute, useful, and necessary caregivers. They also feel not well recognized by other professionals and identify an unspecific view of the nurse by the health care users. Nurses work in a complex context and identify their recognition by the community, despite the lack of conditions to perform their work. Therefore, the professional identity of the nurse if formed by reflections of self-image, hetero-image, and images of reality put together.

KEYWORDS: Nurse's role; Professional competence; Occupational health.

\section{LA IDENTIDAD PROFESIONAL DE LOS ENFERMEROS DE SERVICIOS DE SALUD MUNICIPAL}

RESUMEN: Este estudio objetiva presentar la percepción de los enfermeros de los Servicios de Salud del estado de Rio Grande do Sul/Brasil acerca de su identidad profesional. Se trata de una investigación cualitativa, con la utilización de un cuestionario para la recolección de los datos. Participaron 29 enfermeros de los Servicios de Salud. Para verificación de los datos fue utilizada la técnica del análisis temático y los mismos organizados en torno a tres ejes: autoimagen, heteroimagen e imagen de la realidad. Como resultados, los enfermeros se ven como cuidadores resolutivos, útiles y necesarios en la asistencia. Se sienten poco reconocidos por otros profesionales y señalan una visión inexacta del enfermero por parte de los usuarios. Los enfermeros están inseridos en un contexto complejo y distinguen el reconocimiento de la comunidad, pese a la falta de condiciones para realizar su trabajo. Por lo tanto, la identidad profesional del enfermero se constituye por reflejos de la autoimagen, heteroimagen y de la imagen de la realidad; consustanciados.

PALABRAS CLAVE: Rol del profesional de Enfermería; Competencia profesional; Salud del trabajador.

${ }^{1}$ Enfermeira. Doutora em Enfermagem. Professor Associado do Departamento de Enfermagem da Universidade Federal Santa MariaUFSM. Membro do Grupo de Pesquisa Trabalho, Saúde, Educação e Enfermagem da UFSM.

${ }^{2}$ Acadêmica do Curso de Enfermagem da UFSM. Membro do Grupo de Pesquisa Trabalho, Saúde, Educação e Enfermagem da UFSM. ${ }^{3}$ Enfermeira. Mestranda em Enfermagem na UFSM. Membro do Grupo de Pesquisa Trabalho, Saúde, Educação e Enfermagem da UFSM.

${ }^{4}$ Enfermeira do Hospital Universitário de Santa Maria. Mestre em Enfermagem-UFSM. Membro do Grupo de Pesquisa Trabalho, Saúde, Educação e Enfermagem da UFSM.

${ }^{5}$ Enfermeira. Doutora em Enfermagem. Professor Adjunto do Departamento de Enfermagem da UFSM. Membro do Grupo de Pesquisa Trabalho, Saúde, Educação e Enfermagem da UFSM.

${ }^{6}$ Enfermeira. Membro do Grupo de Pesquisa Cuidado, Saúde e Enfermagem da UFSM. Bolsista de iniciação científica CNPq.

Autor correspondente

Carmem Lúcia Colomé Beck

Rua Coronel Estácio Lemas, 154 - 97020-150 - Santa Maria-RS

E-mail: carmembeck@smail.ufsm.br 


\section{CONSIDERAÇÕES INICIAIS}

A identidade do ser humano é formada e transformada continuamente e esse caráter de (re) construção constante faz com que permaneça sempre incompleta ${ }^{(1)}$. Dessa forma, a identidade é construída na relação do homem com ele mesmo, com os outros e com a sociedade em que está inserido ${ }^{(2)}$.

Nessa relação, destaca-se a associação relacionada ao trabalho que, na vida do homem, cumpre as finalidades essenciais de reprodução social e de expressão, como sujeito. A reprodução social implica na possibilidade de satisfazer necessidades a partir da aquisição de bens de consumo, sendo objetivada por meio do salário pago ao trabalhador. Já a sua expressão como sujeito, envolve a procura do prazer e da satisfação, realçando suas potencialidades como ser humano. Assim, o trabalho fornece uma identidade ao trabalhador que, de outra maneira, nem sempre consegue alguma forma de se mostrar existindo e se relacionando ${ }^{(3)}$.

Quanto à enfermagem, alguns autores afirmam que seu trabalho encontra-se na interface com outros trabalhos na área da saúde e possui características que o identificam, bem como a seus trabalhadores ${ }^{(4)}$. Nesse contexto, em muitas situações a sociedade não faz uma diferenciação entre o enfermeiro e os membros da equipe de enfermagem ${ }^{(5)}$ o que pode apresentar reflexos nas representações, na autoimagem e na auto (des)valorização dos trabalhadores acerca de seu trabalho e das suas funções sociais ${ }^{(2)}$.

Sabe-se que a valorização e o reconhecimento profissional da enfermagem são desafios a serem enfrentados e que estão ancorados em resquícios históricos, e em outros componentes sócio-políticos, os quais estruturam o papel social estabelecido para os enfermeiros na atualidade.

Os estudos acerca da identidade profissional da enfermagem ainda são escassos e buscam explicar a complexidade que permeia a sua construção em diversas realidades históricas, sociais e culturais. Além disso, evidenciam-se desafios profissionais para a enfermagem que se reconfiguram ao longo do tempo, sem serem completamente ultrapassados, tendo como exemplos a falta de reconhecimento social, a baixa remuneração, as longas jornadas de trabalho e a pouca autonomia profissional.

Em relação aos Serviços de Saúde, observase, empiricamente, na realidade de um município do interior do Estado do Rio Grande do Sul (RS), o acúmulo de atribuições do enfermeiro, o que pode comprometer a identificação do seu papel profissional por parte dos usuários e trabalhadores de outras áreas e repercutir negativamente na sua identidade profissional. Diante do exposto, esta pesquisa tem como objetivo conhecer a percepção dos enfermeiros dos Serviços de Saúde de um município do interior do Estado do RS, acerca de sua identidade profissional.

\section{PERCURSO METODOLÓGICO}

Este estudo se caracteriza como pesquisa da área humano-social, do tipo exploratório-descritiva com trajetória metodológica de pesquisa qualitativa.

A população deste estudo foram 29 enfermeiros que exerciam atividades nos Serviços de Saúde vinculados à prefeitura municipal de um Município do interior do estado do RS, que compreende os seguintes serviços: unidades básicas de saúde, unidades de estratégia de saúde da família, serviços especializados e complementares, serviço de vigilância à saúde e coordenadoria do meio ambiente e saneamento.

Para a coleta dos dados, utilizou-se um questionário com questões fechadas referentes a dados sóciodemograficos dos enfermeiros investigados e questões abertas que contemplavam os dados subjetivos do estudo. $\mathrm{O}$ instrumento foi previamente validado em estudo piloto junto a três enfermeiros que atuavam em Serviços de Saúde do Município pesquisado.

Os critérios de inclusão dos participantes do estudo foram: ser enfermeiro; atuar nos serviços mencionados, independentemente do vínculo empregatício (enfermeiros efetivos do município ou com contrato temporário). Assim, todos os sujeitos (52) que atenderam aos critérios de inclusão, receberam o questionário, e desses 29 foram devolvidos, os quais compuseram este estudo.

Os pesquisadores realizaram um primeiro contato com os sujeitos, pessoalmente, no local de trabalho dos enfermeiros e, nesse momento, formalizaram o convite para a participação no estudo, explicaram os objetivos da pesquisa e apresentaram o Termo de Consentimento Livre Esclarecido. Após o consentimento do participante, procedeu-se a entrega do instrumento de pesquisa e estabeleceu-se uma data para devolução do mesmo.

A coleta dos dados ocorreu de março a julho de 2007 e, após o término, os dados obtidos foram identificados, utilizando-se números arábicos antecedidos pela letra "E" (Enfermeiro) de modo a preservar a identidade dos sujeitos pesquisados (E1, E2, E3...).

Foram respeitados os preceitos da ética em 
pesquisa conforme a Resolução n 196/96(6), e o projeto foi autorizado pelo Comitê de Ética e Pesquisa, sob o número do CAEE (Certificado de Apresentação para Apreciação Ética) 0153.0.243.000-06 da Universidade Federal de Santa Maria.

Dentre as modalidades de análise de conteúdo, optou-se pela análise temática embasado na regularidade das afirmações denotando, dessa forma, estruturas de relevância, valores de referência e comportamentos presentes ou subjacentes nos discursos ${ }^{(7)}$.

Inicialmente, procedeu-se a leitura dos dados na íntegra para apreensão inicial de seus conteúdos e, seqüencialmente, estabeleceu-se que os dados seriam analisados em torno de dois eixos temáticos: autoimagem e heteroimagem ${ }^{(5)}$. No decorrer da análise, observou-se que os dados contemplavam um terceiro eixo temático que refletia a imagem da realidade.

Após a leitura exaustiva do material, foram identificados e agrupados os dados que se repetiam e/ ou possuíam semelhança semântica nos diferentes fragmentos em relação aos eixos temáticos supracitados, de acordo com o objetivo do estudo. Posteriormente, foi realizada a categorização dos elementos constitutivos de cada eixo, destacando-se que no decorrer da análise foi identificada a repetição e homogeneidade dos dados.

Os resultados deste estudo foram apresentados a partir das falas que exemplificam as categorias temáticas e organizadas em Quadros, cuja estruturação permite a visualização, facilitando a compreensão das categorias que compõem cada eixo temático.

\section{APRESENTAÇÃO DOS RESULTADOS}

Os sujeitos investigados neste estudo foram, predominantemente, do sexo feminino $(96,4 \%)$, com idade entre 36 e 45 anos (37,3\%), com curso de pósgraduação (88,9\%), sendo a maior parte em Saúde Pública, Coletiva ou Saúde da Família (68,2\%).

Tendo-se em vista o pressuposto de que o trabalho é uma espécie de espelho em que o homem se projeta e se enxerga no mundo, o que confere uma identidade ao trabalhador ${ }^{(8)}$, os dados subjetivos foram analisados em torno dos eixos temáticos: auto-imagem, heteroimagem e imagem da realidade.

A Auto-imagem ${ }^{(5)}$ corresponde à forma como os sujeitos se definem como enfermeiros sendo que, a partir da análise do material, emergiram três categorias: útil e necessário; resolutivo e cuidador conforme Quadro 1.

Quadro 1 - Categorias do eixo temático auto-imagem

\begin{tabular}{|ll|}
\hline $\begin{array}{l}\text { Eixo: auto-imagem } \\
\text { Categorias }\end{array}$ & $\begin{array}{l}\text { Fragmento } \\
\text { Útil e necessário }\end{array}$ \\
& $\begin{array}{l}\text { [...] eu sinto que sou útil e } \\
\text { necessária para os usuários que } \\
\text { freqüentam a unidade básica ( E.1). }\end{array}$ \\
Resolutivo & $\begin{array}{l}\text { [...] procuro solucionar um problema } \\
\text { da forma mais correta [...] (E.2). }\end{array}$ \\
Cuidador & $\begin{array}{l}\text { Tenho a imagem de uma cuidadora } \\
\text { que acredita no que faz (E.24). }\end{array}$ \\
\hline
\end{tabular}

A imagem de útil e necessário refletiu-se sob diversos prismas e em diferentes intensidades. Os sujeitos consideraram seu trabalho importante e foram unânimes ao afirmar que são reconhecidos e valorizados pelos usuários, que demonstram afeto pelo trabalhador e depositam confiança em seu trabalho.

A imagem de resolutivo reflete o enfermeiro como trabalhador participativo, crítico-reflexivo e comunicativo, que desenvolve seu trabalho com comprometimento, dedicação, responsabilidade, ética e em parceria com trabalhadores de outras áreas da saúde, visando a resolução dos problemas da comunidade e prestação de assistência integral.

$\mathrm{Na}$ imagem de cuidador, o enfermeiro se considera empático e afetivo, projetando-se nos usuários e os assistindo de forma carinhosa e acolhedora. Além disso, ao se definirem como cuidadores os enfermeiros afirmaram que essa condição faz com que se sintam motivados em seu trabalho e confiantes na efetividade de suas ações.

O segundo eixo temático evidencia a hetoroimagem que, de acordo com alguns autores ${ }^{(5)}$, reflete a forma como os sujeitos acreditam que os outros profissionais e a comunidade veem a sua prática. A partir desse entendimento, construíram-se três categorias temáticas: conforme Quadro 2.

Quadro 2 - Categorias do eixo temático heteroimagem

\begin{tabular}{|c|c|}
\hline \multicolumn{2}{|l|}{ Eixo: heteroimagem } \\
\hline Categorias & Fragmentos \\
\hline $\begin{array}{l}\text { Ser referência na } \\
\text { unidade }\end{array}$ & $\begin{array}{l}\text { A procura por mim; querem } \\
\text { agendar consulta comigo e } \\
\text { retornam }[\ldots](\text { E.4). }\end{array}$ \\
\hline $\begin{array}{l}\text { Visão inespecífica } \\
\text { do enfermeiro }\end{array}$ & $\begin{array}{l}\text { Estar sempre resgatando o trabalho } \\
\text { do enfermeiro, tentando provar que } \\
\text { tu és capaz, [...] muitas vezes, um } \\
\text { quebra-galho de médico (E.3). }\end{array}$ \\
\hline $\begin{array}{l}\text { Pouco } \\
\text { reconhecimento por } \\
\text { outros profissionais }\end{array}$ & $\begin{array}{l}\text { Gosto do que faço, porém gostaria } \\
\text { de ser mais reconhecida por outros } \\
\text { profissionais }[\ldots](\text { E. } 10) \text {. }\end{array}$ \\
\hline
\end{tabular}


Ser referência na unidade é uma imagem que se refletiu de forma intensa, pois os enfermeiros afirmaram que os usuários procuram primeiramente a figura do enfermeiro no Serviço de Saúde, seguem suas orientações e participam das atividades propostas, o que confere satisfação aos trabalhadores.

Apesar de ser referência, os usuários possuem uma visão inespecífica do enfermeiro, na medida em que desconhecem o papel que esse desempenha, chegando a confundir as atividades do enfermeiro com as realizadas por outros trabalhadores. Nesse sentido, há desapontamento por parte dos enfermeiros, pela necessidade de reafirmar o seu trabalho, com suas competências específicas para não ser confundido com “quebra-galho" de outros trabalhadores.

Soma-se a isso o fato de o enfermeiro ser pouco reconhecido por outros trabalhadores da área da saúde que muitas vezes desconhecem e não valorizam as suas atividades privativas.

O eixo temático a imagem da realidade reflete como os enfermeiros percebem seu contexto de trabalho no que se refere aos Serviços de Saúde e à comunidade por eles assistida. Com relação a esse eixo, os fragmentos convergiram para duas categorias: falta de condições para realizar um bom trabalho e reconhecimento da comunidade, conforme ilustra o Quadro 3.

Quadro 3 - Categorias do eixo temático imagem da realidade

\section{Eixo: imagem da realidade \\ Categorias}

Falta de condições para realizar um bom trabalho
Fragmentos

Falta de determinados materiais, equipamentos, medicamentos, problemas de comunicação entre gestão/unidades e também intersetoriais; vínculo empregatício instável, ausência de funcionários [...](E.6).

Reconhecimento da [...] vejo que a comunidade comunidade também retribui valorizando, respeitando e reconhecendo $o$ trabalho com dedicação [...] (E.1).

Os enfermeiros são unânimes em relação à falta de condições para realizar um bom trabalho, relacionado a diversos fatores como: carência de recursos materiais e de pessoal; dificuldades de comunicação entre os Serviços de Saúde; entraves burocráticos e políticos em que os enfermeiros apontam a descontinuidade de idéias e projetos.

Outro reflexo da falta de condições para realizar um bom trabalho é a incompreensão, por parte de alguns usuários, da falta de medicamentos e carência de médicos especialistas nos Serviços de Saúde do Município. Os enfermeiros afirmaram que a desinformação em relação à rotina de prioridade de atendimento da população e situação que o enfermeiro vivencia diariamente repercute no descontentamento da população e no modo como ela percebe o serviço.

Por outro lado, os trabalhadores se referem ao reconhecimento da comunidade e o exemplificam com a aceitação do serviço, do estabelecimento de vínculo com a mesma, demonstração de afeto e gratidão com os membros da equipe, e enfatizam, ainda, a adesão dos usuários às orientações e condutas propostas pelo enfermeiro.

Em relação aos colegas de trabalho, houve manifestações positivas, destacando a qualificação da equipe, o bom relacionamento interpessoal e com a comunidade, além do comprometimento da maioria dos trabalhadores com o serviço.

\section{DISCUSSÃO DOS RESULTADOS}

Os resultados deste estudo evidenciam uma população predominantemente do sexo feminino, o que reproduz a realidade da maioria dos serviços de saúde e nos cursos de graduação em enfermagem, em que o número de acadêmicos do sexo feminino ultrapassa $85 \%{ }^{(9)}$.

A questão do gênero é relevante ao se abordar a identidade profissional uma vez que remete a contextos sócio-culturais de diferentes momentos históricos que repercutem nas representações sociais da profissão. Exemplo disso é um estudo etnográfico ${ }^{(10)}$ de textos produzidos por enfermeiras na modernidade que atribui à posição feminina da profissão, grande parte de dilemas como crise de competência, vocação e identidade.

Sobre o eixo temático auto-imagem, a imagem de útil reflete que os enfermeiros investigados se consideram sujeitos fundamentais para a prestação da assistência nos serviços em que atuam. Esse dado, por um lado, pode representar satisfação profissional considerando-se que se sentir útil remete a valorização do trabalho; por outro, pode constituir-se uma estratégia defensiva do trabalhador que diante de situações geradoras de sofrimento, como o excesso de atividades, grande demanda de pacientes e 
condições inadequadas de trabalho, procura doar-se ao máximo na tentativa de "compensar” tais situações.

Um estudo sobre a construção da identidade do enfermeiro $^{(11)}$ aponta que o trabalho preenche importantes parcelas na vida dos trabalhadores e pode fazer com que esses se sintam vivos e presentes, e isso implica em imprimir algo de si no cotidiano de trabalho.

Assim, evidencia-se que a auto-imagem útil também reflete que os enfermeiros se sentem vivos e presentes em seu trabalho. Da mesma forma, a autoimagem de resolutivo implica em comprometer-se e (re) pensar o trabalho imprimindo-lhe algo de si.

A auto-imagem de cuidador, refletida neste estudo, caracteriza o enfermeiro e seu foco de trabalho que é o cuidado de enfermagem. Sob esse viés, cuidar em enfermagem significa auxiliar o outro em situações em que ele não consegue satisfazer suas próprias necessidades. Além disso, esse ato envolve disciplina, conhecimento, paciência, espaço, vivência, dedicação, ciência e disposição para que possa ser realizado ${ }^{(12)}$.

A imagem de cuidador também pode remeter a concepção histórica da enfermagem, quando assume o caráter caritativo, o que dificulta a proposição de ações efetivas no campo da saúde do trabalhador, uma vez que, esquece-se que a profissão de enfermagem é efetivada em uma sociedade concreta, com carências, e que os trabalhadores vendem sua força de trabalho para garantir sua existência ${ }^{(13)}$.

No eixo temático heteroimagem, a visão inespecífica do enfermeiro por parte dos usuários refletida neste estudo, é uma questão preocupante que foi evidenciada em outro estudo sob outro prisma da auto-imagem dos enfermeiros em Saúde Pública ${ }^{(5)}$. Assim, observa-se que os resultados são convergentes e ratificam a premissa de que a construção da identidade do enfermeiro ocorre pela relação do ser com o outro, em que o outro diz e reafirma quem é o enfermeiro, constituindo-se, assim, o seu espelho que reflete a heteroimagem ${ }^{(11)}$.

O enfermeiro, por vezes, desempenha atividades que são da competência de outros profissionais, o que contribui para que os usuários não tenham o discernimento de seu papel profissional. Dessa forma, o enfermeiro apreende estas atividades para si, o que dificulta a especificidade de suas ações e a delimitação do seu papel, tendendo a minimizar sua visibilidade diante da instituição, da equipe de saúde e dos usuários ${ }^{(5)}$.

Nos Serviços de Saúde esse fato é evidente, pois o enfermeiro é visto como referência na unidade pelos usuários e é procurado com freqüência. Apesar disso, o enfermeiro não se sente valorizado pelos demais trabalhadores da equipe de saúde o que é ratificado em outro estudo ${ }^{(5)}$.

A imagem da realidade, além do reconhecimento da comunidade, refletiu a falta de condições para realizar um bom trabalho, condições essas caracterizadas pela falta de recursos humanos, precariedade nos recursos físicos e déficit de recursos materiais. Sobre isso, estudo aponta que um ambiente de trabalho com excesso de condições negativas, como a escassez de recursos materiais e trabalhadores em número reduzido, podem desencadear tensões e prejudicar o ser humano ${ }^{(14)}$.

Outro estudo(15) encontrou resultados semelhantes e afirma que os trabalhadores devem assumir uma postura crítica com relação às condições precárias de trabalho decorrente da situação adversa do sistema de saúde.

Os resultados deste estudo revelam que apesar da incompreensão de alguns usuários e da carência de reconhecimento de outros profissionais, os enfermeiros sentem-se reconhecidos e valorizados pela comunidade. Essa contradição também foi encontrada em uma investigação com enfermeiros ${ }^{(11)}$, em que se evidenciou a satisfação individual dos trabalhadores, apesar dos conflitos no trabalho.

Neste entendimento, o reconhecimento não é uma reivindicação secundária dos trabalhadores, mostrando-se decisiva na dinâmica da mobilização subjetiva da inteligência e da personalidade no trabalho, o que é designado na psicologia e psicodinâmica do trabalho como "motivação no trabalho". Assim, quando o reconhecimento passa despercebido em meio à indiferença geral ou é negado por outros, acarreta em sofrimento para os trabalhadores ${ }^{(16)}$.

Estudo sobre psicodinâmica do trabalho ${ }^{(17)}$ afirma que o reconhecimento proporciona ao trabalhador prazer e auto-realização, os quais se dão por meio da valorização do esforço e do sofrimento empregados na realização do trabalho. Dessa forma, o reconhecimento possibilita uma atribuição de sentido a essas vivências dos trabalhadores, possibilitando ao sujeito a construção de sua identidade.

\section{ALGUMAS CONSIDERAÇÕES}

Este estudo reafirma a evidência que a identidade profissional do enfermeiro é formada por reflexos da auto-imagem, heteroimagem e imagem da realidade consubstanciados.

Os enfermeiros dos Serviços de Saúde 
investigados neste estudo, percebem-se como cuidadores resolutivos e necessários, o que aponta para uma auto-imagem positiva.

No que se refere a heteroimagem, os enfermeiros refletiram uma percepção ambígua em que, apesar de serem referência nos serviços em que atuam, sentemse pouco reconhecidos por outros profissionais, e percebem a visão inespecífica do enfermeiro por parte dos usuários, o que pode repercutir negativamente em sua identidade profissional

Os enfermeiros vivenciam um contexto laboral dinâmico, complexo e por um lado, constatam a falta de condições para realizar o trabalho, o que lhes causa insatisfação e os expõe a incompreensão de alguns pacientes. Por outro, percebem o reconhecimento da comunidade, mesmo diante das dificuldades encontradas e da consciência de que poderiam fazer de outra forma o seu trabalho.

Assim, a heteroimagem e a imagem da realidade remetem a desafios que se reconfiguram ao longo da história da enfermagem como profissão. Assim, para transpor essa realidade, é necessário que o enfermeiro tenha clareza da importância de delimitar suas ações em seu campo de atuação, desempenhando as atividades de sua competência e reivindicando a revisão e possível reconstrução do seu processo de trabalho.

Como limitações do estudo, menciona-se a complexidade do objeto de estudo, a identidade profissional, bem como o fato de retratar a especificidade de um município do Rio Grande do Sul, considerandose as peculiaridades das diferentes regiões do país.

As limitações e as possíveis transformações desejadas, suscitam novas investigações acerca da identidade profissional de acadêmicos de enfermagem, a fim de observar as repercussões da formação dos mesmos no percurso da graduação.

\section{REFERÊNCIAS}

1. Hall S. A identidade cultural na Pós-modernidade. $4^{\text {a }}$ ed. Rio de Janeiro: DPeA; 2000.

2. Oliveira BGRB de. A passagem pelos espelhos: a construção da identidade profissional da enfermeira. Texto Contexto Enferm. 2006; 15(1):60-7.

3. Gonzáles RMB, Beck CLC. O sofrimento e o prazer no cotidiano dos trabalhadores de enfermagem. Texto Contexto Enferm. 2002 Jan/Abr; 11(1):169-86.

4. Silva, AL, Padilha MIC de S, Borestein MS. Imagem e Identidade profissional na construção do conhecimento em enfermagem. Rev Latino-Am Enferm. 2002 Jul/Ago;
10(4):586-95.

5. Gomes AMT, Oliveira DC de. A auto e hetero-imagem do enfermeiro em saúde pública: um estudo de representações sociais. Rev Latino-Am Enferm. 2005 Nov/Dez; 13(6):1001-8.

6. Brasil. Conselho Nacional de Saúde (BR). Resolução 196, de 10 de outubro de 1996 que Aprova as diretrizes e normas regulamentadoras de pesquisas envolvendo seres humanos. [página na internet] [2002 Jul 12]. Disponível: http://conselho.saude.gov.br/resolucoes/ 1996/Reso196.doc

7. Minayo MCS. O desafio do conhecimento: pesquisa qualitativa em saúde. 10ª ed. São Paulo: Hucitec; 2007.

8. Codo W. O que é alienação. $9^{a}$ ed. São Paulo: Brasiliense; 1994.

9. Ojeda BS, Eidt OR, Canabarro S, Corbellini VL, Creutzberg M. Saberes e verdades acerca da enfermagem: discursos de alunos ingressantes [periódico na Internet]. Rev Bras Enferm. 2008 [acesso em 2009 Mar 31] 61(1):78-84. Disponível: www.scielo.br

10. Moreira MCN. Imagens do espelho de Vênus: mulher, enfermagem e modernidade. Rev Latino-Am Enferm. 1999 Jan; 7(1):55-65.

11. Netto LFS de A, Ramos FRS. Considerações sobre o processo de construção da identidade do enfermeiro no cotidiano de trabalho. Rev Latino-Am Enferm. 2004 Jan/Fev; 12(1):50-7.

12. Sanches MO, Pedro ENR. Ações e expressões de cuidado na prática educativa de enfermeiros docentes. Rev Gaúcha Enferm. 2008; 29(1):11-7.

13. Rodrigues RM. Enfermagem compreendida como vocação e sua relação com as atitudes dos enfermeiros frente às condições de trabalho. Rev Latino-Am Enferm. 2001 Nov/Dez; 9(6):76-82.

14. Thofehrn MB, Amestoy SC, Carvalho KK DE.; Andrade, F. P.; Milbrath, V. M. Assédio moral no trabalho da enfermagem. Cogitare Enferm. 2008; 13(4):597-601.

15. Porto IS. Identidade da enfermagem e identidade profissional da enfermeira: tendências encontradas em produções científicas desenvolvidas no Brasil. Esc Anna Nery Rev Enferm. 2004; 8(1):92-100.

16. Dejours C. A banalização da injustiça social. Rio de Janeiro: Fundação Getúlio Vargas; 1999.

17. Mendes AM. Psicodinâmica do trabalho: teoria, método e pesquisas. São Paulo: Casa do Psicólogo; 2007. 